Fermilab TM-2235

\title{
A modified post damping ring bunch compressor beamline for the TESLA linear collider
}

\author{
P. Piot \\ Fermi National Accelerator Laboratory, Batavia IL 60510, USA \\ W. Decking \\ Deutsches Elektronen-Synchrotron, Notkestraße 85, 22603 Hamburg, Germany
}

March 23, 2004

\begin{abstract}
We propose a modified bunch compressor beamline, downstream of the damping ring, for the TESLA linear collider. This modified beamline uses a third harmonic radio-frequency section based on the $3.9 \mathrm{GHz}$ superconducting cavity under development at Fermilab. In our design the beam deceleration is about $\sim 50 \mathrm{MeV}$ instead of $\sim 450 \mathrm{MeV}$ in the original design proposed in Reference [W. Decking et al., report TESLA-2000-40, DESY Hamburg (2000)].
\end{abstract}

\section{Introduction}

The equilibrium bunch length in the TESLA damping ring is approximately $6 \mathrm{~mm}$ (rms). A bunch compressor beamline is therefore needed to compress the bunch, by a factor 20, down to the requested bunch length of $0.3 \mathrm{~mm}(\mathrm{rms})$. In the TESLA linear collider [1] the main components of the compression beamline are: a radio-frequency (rf) section and a magnetic bunch compressor (the beamline also incorporates a spin rotator and a skew correction section, see Reference [2] for details). The rf-section is tuned to impart the proper time-energy correlation along the bunch, as required for bunch compression in a downstream wiggler-type magnetic bunch compressor. As studied in References [2] and [3], efficient bunch compression in TESLA linear collider and TESLA X-FEL is achieved by correcting distortions of the longitudinal phase space prior to bunch compression. Such a correction process is especially important when the incoming bunch length is not negligible compared to the inverse of the rf-wavenumber. In this latter case the longitudinal phase space accumulates nonlinearities induced by the $\mathrm{rf}$ cos-like time-dependence. To correct for these longitudinal phase space distortions, it is proposed in Reference [2], to operate the $(1.3 \mathrm{GHz})$ rf-section downstream of the bunch compressor at a decelerating phase. In the context of the X-ray FEL, in Reference [3], it is instead suggested to introduce an higher harmonic rf-field $(3.9 \mathrm{GHz})$ to correct for the rf-induced distortion coming from the $1.3 \mathrm{GHz}$ rf-section. The goal of the present note is to discuss the use of this latter correction scheme in the TESLA linear collider bunch compression beamline. 


\section{Comparison of the two correction processes}

Let's first review analytically the two methods mentioned in Section 1. Consider the incoming longitudinal phase space downstream to have no correlation, i.e. of the form $E_{0}(s)=E_{0}+$ $\mathcal{E}(s)$, wherein $\mathcal{E}(s)$ represent the stochastic energy spread. Downstream of an rf-section of frequency $f$, operated at the phase $\varphi_{1}$, and accelerating voltage $V_{1}$, we have for the fractional energy spread:

$$
\delta\left(s_{0}\right) \simeq \frac{-k V_{1} \sin \left(\varphi_{1}\right)}{E} s_{0}+\frac{-k^{2} V_{1} \cos \left(\varphi_{1}\right)}{2 E} s_{0}^{2} \doteq \alpha s_{0}+\beta s_{0}^{2},
$$

wherein $k=c / f$ is the rf-wavenumber ( $c$ being the velocity of light), $E=E_{0}+V_{1} \cos \left(\varphi_{1}\right)$ is the energy downstream of the rf-section. We have assumed the stochastic energy spread to be much smaller than the induced energy spread, typically $|\mathcal{E}| \ll\left|V_{1} \cos \varphi_{1}\right|$. Downstream of a bunch compressor characterized by the usual linear and second order momentum compactions ${ }^{1}, R_{56}$ and $T_{566}$ respectively, the electron longitudinal position $s_{0}$ is mapped to $s_{2}$ via:

$$
s_{2}=s_{0}+R_{56} \delta\left(s_{0}\right)+T_{566} \delta^{2}\left(s_{0}\right)
$$

Thus the amount of compression is given by the ratio:

$$
\frac{s_{2}}{s_{0}}=\left(1+\alpha R_{56}\right)+s_{0}\left(\alpha^{2} T_{566}+\beta R_{56}\right) \doteq \mathcal{C}+s_{0}\left(\alpha^{2} T_{566}+\beta R_{56}\right)
$$

Given the compressor parameters, and the desired compression factor, the rf-parameters should be set to achieve the required value for $\mathcal{C}$ while simultaneously canceling the second term of the latter equation r.h.s. Such requirements yield the system of two equations with two unknowns,

$$
V_{1} \sin \left(\varphi_{1}\right)=\frac{-\kappa E_{0}}{1-\rho \kappa^{2}}, \text { and } V_{1} \cos \left(\varphi_{1}\right)=\frac{\rho \kappa^{2} E_{0}}{1-\rho \kappa^{2}},
$$

wherein $^{2} \kappa \doteq(\mathcal{C}-1) /\left(k R_{56}\right)$ and $\rho \doteq 2 T_{566} / R_{56}$. The solution is:

$$
\tan \left(\varphi_{1}\right)=\frac{-1}{\rho \kappa}=\frac{-k R_{56}^{2}}{2(\mathcal{C}-1) T_{566}}, \text { and } V_{1}=\frac{\kappa E_{0} \sqrt{1+\rho \kappa^{2}}}{\left|1-\rho \kappa^{2}\right|}
$$

Because $V_{1} \cos \left(\varphi_{1}\right) \propto T_{566} / R_{56}^{3}$ along with the fact that $\operatorname{sign}\left(T_{566} / R_{56}\right)=-1$ for a wiggler bunch compressor, the beam is decelerated during the correction process. This is the technique used in Reference [2].

\footnotetext{
${ }^{1}$ In our convention $R_{56}<0$ for wiggler compressor: electron in the head of the bunch corresponds to negative time.

${ }^{2}$ the definition of the constants $\kappa$ and $\rho$ yield: $\kappa>0, \rho<0$. Note that for a wiggler-type bunch compressor one has $\rho \simeq-3$
} 
The alternative technique of introducing a third harmonic field [3], with accelerating voltage $V_{3}$ and phase $\varphi_{3}$, is simply analyzed by noting that now

$$
\alpha=\frac{-k V_{1} \sin \left(\varphi_{1}\right)-3 k V_{3} \sin \left(\varphi_{3}\right)}{E^{\prime}}, \text { and } \beta=\frac{-k^{2} V_{1} \cos \left(\varphi_{1}\right)-9 k^{2} V_{3} \cos \left(\varphi_{3}\right)}{E^{\prime}},
$$

wherein $E^{\prime}=E_{0}+V_{1} \cos \left(\varphi_{1}\right)+V_{3} \cos \left(\varphi_{3}\right)$. Compared to Reference [3], the system is underconstrained. In order to have an independent control of the two terms in Eq. 3, we set $\varphi_{1}=-\pi / 2$ and $\varphi_{3}=\pi$ and accordingly calculate the required accelerating voltages:

$$
V_{1}=\frac{9 \kappa E_{0}}{9-\rho \kappa^{2}}, \text { and } V_{3}=-\frac{\rho \kappa^{2} E_{0}}{9-\rho \kappa^{2}}
$$

Similarly to the first technique, the beam has to be decelerated during this alternative correction process. However by comparing the second of Eq. 4 with Eq. 7 we estimate that the beam deceleration induced by the first correction method is approximately 9 times larger than the deceleration induced by the latter approach (since $\left|\rho \kappa^{2}\right| \simeq 0.01 \ll 1$ ).

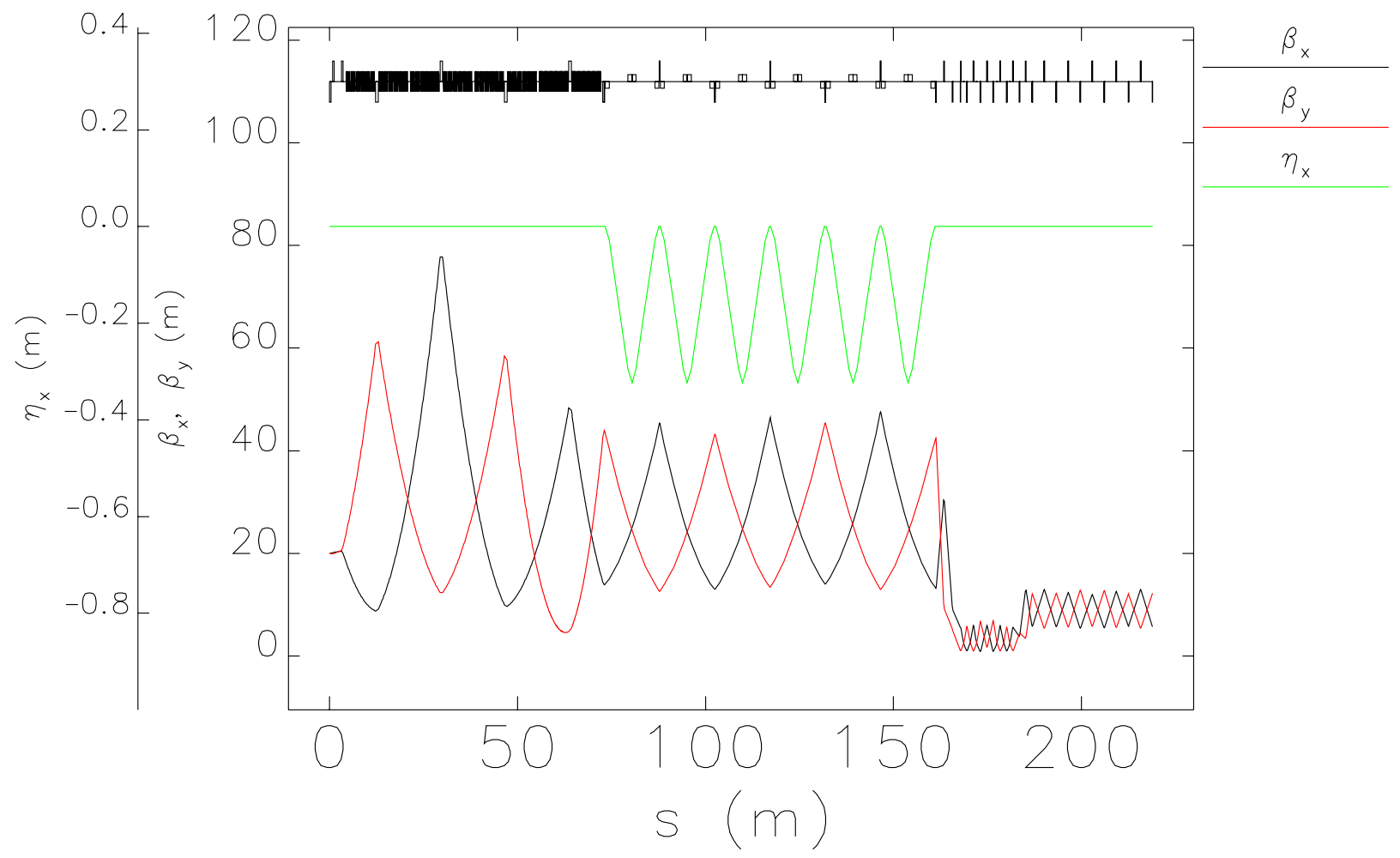

Figure 1: Optical function along the compressor beamline of TESLA linear collider for both option 1 and 2. The rf-section extends from $s \sim 10$ to $\sim 75 \mathrm{~m}$, the wiggler compressor from $\sim 75$ to $\sim 175 \mathrm{~m}$. 


\section{Option 1: The original TESLA bunch compressor}

In the present note we used slightly different parameters for the wiggler compressor compared to Reference [2]. These modified parameters are the results of a more recent optimization [4]. The rf-section comprises four $1.3 \mathrm{GHz}$ accelerating modules shown as $2 \times 4$ black rectangles in Fig. 1. The bunch compressor parameters are gathered in Table 1. The beamline along with the optical functions is shown in Fig. 1. The performance of the present beamline

\begin{tabular}{|c|c|c|c|}
\hline compressor parameters & value & & units \\
\hline wiggler $R_{56}$ & -194.41 & & $\mathrm{~mm}$ \\
\hline wiggler $T_{556}$ & 291.93 & & $\mathrm{~mm}$ \\
\hline input beam parameters & & & units \\
\hline charge & 3.2 & & $\mathrm{nC}$ \\
\hline mean momentum & 5000 & & $\mathrm{MeV} / \mathrm{c}$ \\
\hline rms bunch length & 6000 & & $\mu \mathrm{m}$ \\
\hline rms relative momentum spread & 0.130 & & $\%$ \\
\hline horizontal emittance & 0.8 & & nm-rd \\
\hline vertical emittance & 2.0 & & pm-rd \\
\hline rf-section parameters & option 1 & option 2 & \\
\hline number of $1.3 \mathrm{GHz}$ cavities & 48 & 36 & \\
\hline number of $3.9 \mathrm{GHz}$ cavities & 0 & 12 & \\
\hline $1.3 \mathrm{GHz}$ accel. voltage $V_{1}$ & 19.7 & 25.0 & $\mathrm{MV} / \mathrm{m}$ \\
\hline $1.3 \mathrm{GHz}$ phase $\varphi_{1}$ & -118 & -90 & rf-deg $(1.3 \mathrm{GHz})$ \\
\hline $3.9 \mathrm{GHz}$ accel. voltage $V_{3}$ & - & 14.0 & $\mathrm{MV} / \mathrm{m}$ \\
\hline $3.9 \mathrm{GHz}$ phase $\varphi_{3}$ & - & -180 & rf-deg $(3.9 \mathrm{GHz})$ \\
\hline ouput beam parameters & option 1 & option 2 & \\
\hline mean momentum & 4539.7 & 4948.2 & $\mathrm{MeV} / \mathrm{c}$ \\
\hline rms bunch length & 297.54 & 281.31 & $\mu \mathrm{m}$ \\
\hline rms relative momentum spread & 3.08 & 3.01 & $\%$ \\
\hline horizontal emittance & 0.906 & 0.813 & nm-rd \\
\hline vertical emittance & 2.306 & 2.064 & pm-rd \\
\hline Deceleration $\Delta p$ & 460.30 & 51.80 & $\mathrm{MeV} / \mathrm{c}$ \\
\hline
\end{tabular}

Table 1: Summary table of the beamline and beam parameters for the two options studied in the present notes (see text for details).

was investigated by tracking simulations performed with the computer program elegant [5]. Tracking studies included both incoherent and coherent synchrotron radiation effects in the bending dipoles of the wiggler compressor. The incoming phase space distribution was assumed to be Gaussian in the three degree-of-freedoms, with Twiss parameters: $\beta_{x}=\beta_{y}=$ $20 \mathrm{~m}, \alpha_{x}=\alpha_{y}=0$, and the un-normalized emittances were taken to be $\tilde{\varepsilon}_{x, u}=0.8 \mathrm{~nm}$ and $\tilde{\varepsilon}_{y, u}=2 \mathrm{pm}$, these parameters corresponding to the beam upon ejection from the damping ring. The initial longitudinal phase space is assumed to have no correlation, and its rms bunch length and fractional momentum spread are respectively $\sigma_{z}=6 \mathrm{~mm}$, and $\sigma_{\delta}=1 \times 10^{-3}$. 
The resulting transverse and longitudinal phase spaces downstream of the bunch compressor beamline are presented in Figure 2. The corresponding beam parameters are gathered in Table 1 under the column "option 1".
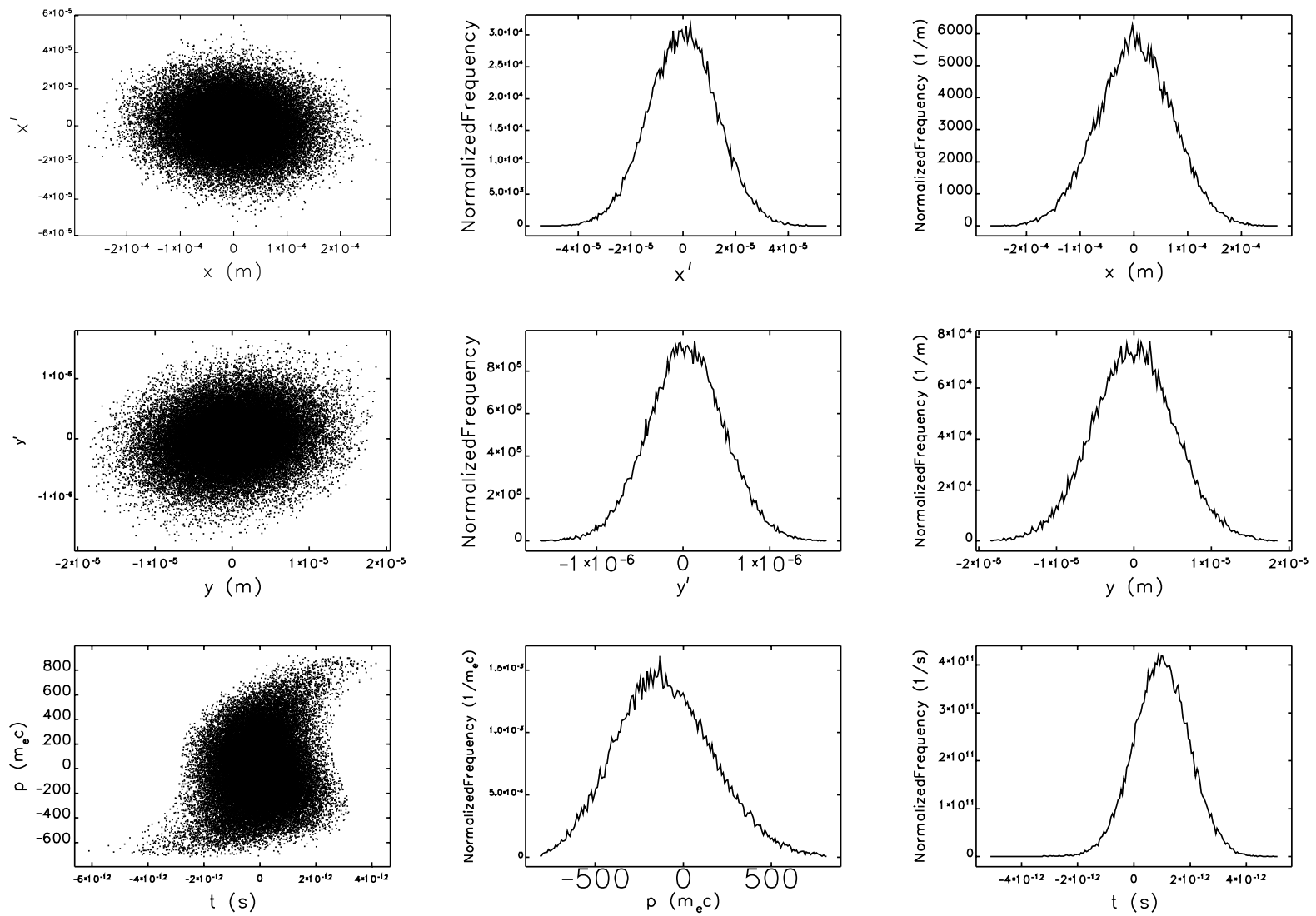

Figure 2: Phase spaces and corresponding projections at the bunch compressor beamline exit for option 1. First, second and third row correspond respectively to horizontal, vertical and longitudinal phase spaces.

\section{Option 2: The modified TESLA bunch compressor}

In the modified beamline, the last $1.3 \mathrm{GHz}$ accelerating module of option 1 (two last black boxes at $s=60 \mathrm{~m}$ in Fig. 1) is replaced by a $3.9 \mathrm{GHz}$ accelerating module consisting of 12 cavities. The cavities are TESLA-type cavities scaled to $3.9 \mathrm{GHz}$ [6]. The same input distribution as the one used for option 1 was used for tracking through the option 2 beamline. It should be noted that in these latter simulations, we did consider collective effects due to geometric wakefield arising in the $3.9 \mathrm{GHz}$ cavities. The $3.9 \mathrm{GHz}$ cavities have smaller aperture than the $1.3 \mathrm{GHz}$ cavities. To estimate the wakefield effects we consider the results presented in Reference [7] where a parametrization for the delta wake-potential of an accelerating module consisting of four $3.9 \mathrm{GHz}$ cavities accelerating was derived. We extend 

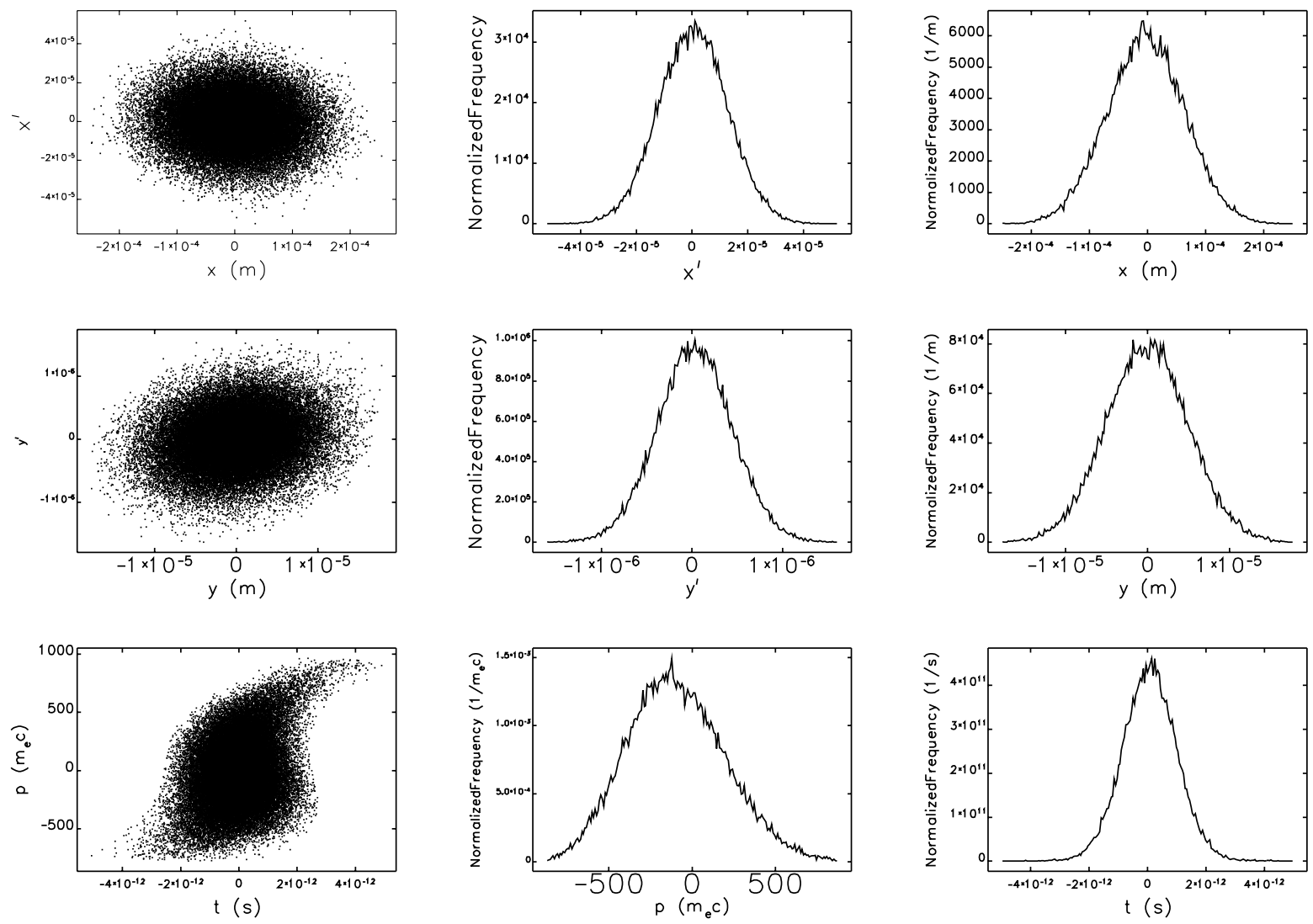

Figure 3: Phase spaces and corresponding projections at the bunch compressor beamline exit for option 1. First, second and third row correspond respectively to horizontal, vertical and longitudinal phase spaces.

this wake-potential to a system of 12 cavities by modifying the wake-potential function as follows:

$$
w_{\|}^{\delta}(s)=-\Theta(s)\left[954 e^{-\sqrt{s / 8.4 .10^{-4}}}+2.7 \frac{\cos \left(5830 s^{0.83}\right)}{\sqrt{s}+195 s}+0.036 \delta(s)\right]
$$

wherein $\Theta(s)$ and $\delta(s)$ are respectively the Heaviside and Dirac function. The units of $w_{\|}^{\delta}$ are $[\mathrm{V} / \mathrm{pC}]$. Our modifications simply consist in scaling the two first terms amplitude by a factor 3 , the factor in front of the $\delta$-function being kept the same as Reference [7], that is we assume that there is only one pair of beam pipe step transition. The latter wakepotential was used in the tracking simulations performed with elegant. As expected from the results presented in Reference [7], the impact on the beam dynamics of the wakefield associated to the $3.9 \mathrm{GHz}$ section was confirmed to be insignificant for the nominal bunch charge and length of respectively $Q=3.2 \mathrm{nC}$ and $\sigma_{z}=6 \mathrm{~mm}$. The phase spaces resulting from tracking, downstream of the bunch compressor beamline, are presented in Figure 3. The corresponding beam parameters are gathered in Table 1 under the column "option 2". 


\section{Conclusion}

We have shown that the inclusion of a third harmonic radio-frequency section downstream of three TESLA accelerating modules reduces substantially the beam deceleration: from $\sim 450 \mathrm{MeV}$, in the original design, to $\sim 50 \mathrm{MeV}$. In the modified beamline, the 12 cavities of each $(1.3 \mathrm{GHz})$ accelerating modules are operated at approximately $25 \mathrm{MV} / \mathrm{m}$, the 12 $(3.9 \mathrm{GHz})$ cavities of the decelerating module operate with approximately $14 \mathrm{MV} / \mathrm{m}$. In the present note we did not present a detailed design of, e.g. the $3.9 \mathrm{GHz}$ decelerating module so that our beamline is exactly the same length of the original beamline. However taking into account the expected performance of the $3.9 \mathrm{GHz}$ cavities, we believe the total length of the bunch compression beamline may be slightly shortened (by approximately $10 \mathrm{~m}$ ). The performance of the modified bunch compressor beamline and original design are very similar as can be seen in Fig. 4 where the longitudinal phase spaces for the two options are compared.
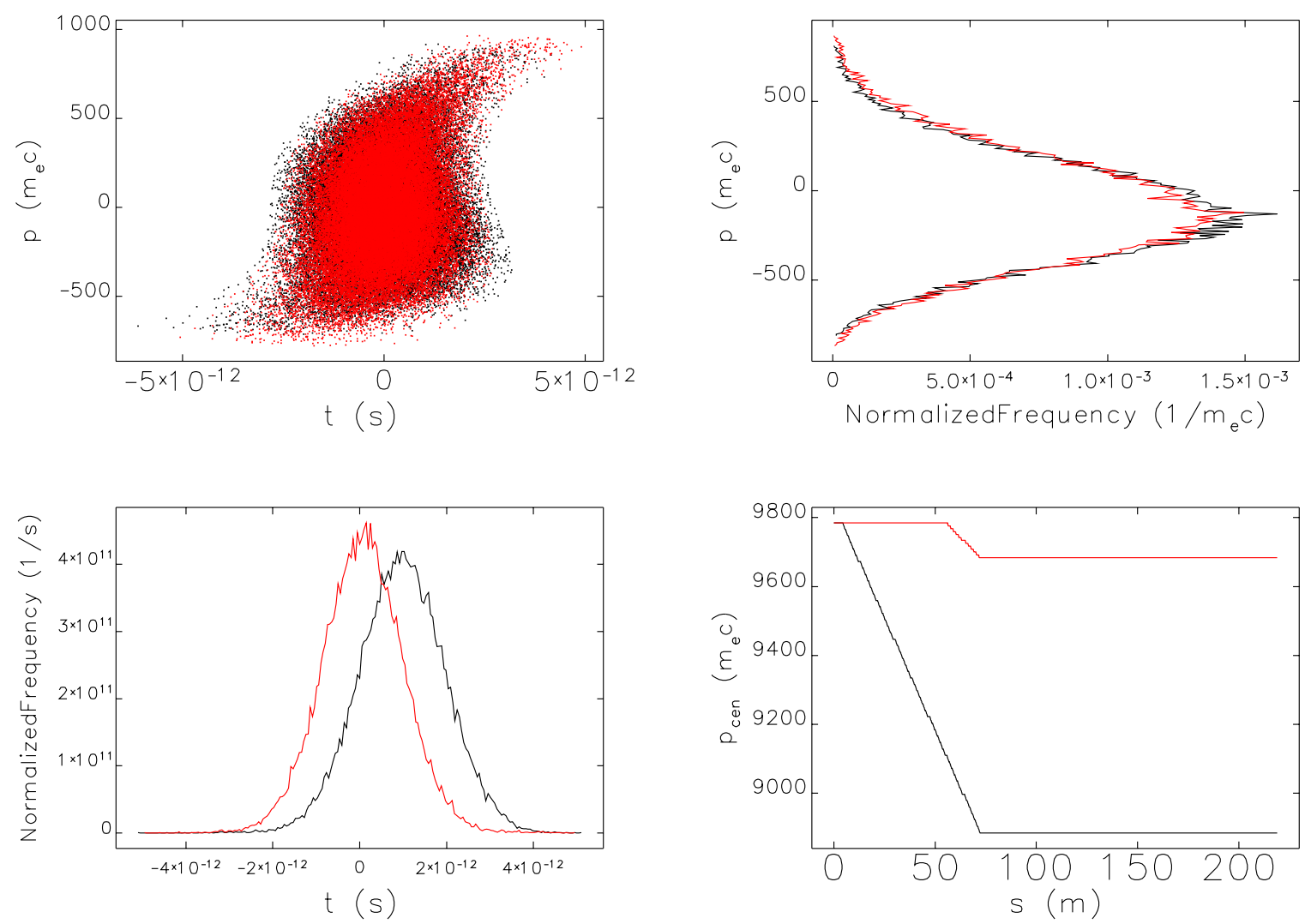

Figure 4: (color) Comparison of the longitudinal phase space (top left), energy spectrum (top right), time density profile (bottom left) and momentum evolution along the beam line (bottom right) between option 1 (black) and option 2 (red).

As a concluding remark, it should be noted that linearizing the longitudinal phase space 
using a third order rf-section before compression is well suited especially if it is desired to compress the bunch to smaller length than $300 \mu \mathrm{m}$. A tighter compression would reduce the impact of transverse wakefields accumulated along the linac and thereby weaken the so-called "banana effect" [8]. In such a case the compression will have to be staged (two stages are likely to be enough).

\section{References}

[1] TESLA, The superconducting electron-positron linear collider with an integrated X-ray laser laboratory, report DESY 2001-011, DESY Hamburg (2001)

[2] W. Decking, G. Hoffstätter, and T. Limberg, "Bunch compressor for the TESLA linear collider", report TESLA-2000-40, DESY Hamburg (2000)

[3] K. Flöttmann, T. Limberg and P. Piot, "Generation of ultra-short electron bunches by cancellation of nonlinear distortions in the longitudinal phase space", report TESLAFEL-2001-06, DESY Hamburg (2001)

[4] W. Decking, MAD input files for the latest TESLA bunch compressor (2004)

[5] M. Borland, "elegant: A Flexible SDDS-Compliant Code for Accelerator Simulation", Advanced Photon Source LS-287, ANL (2000)

[6] T. Khabibouline, N. Solyak and R. Wanzenberg "Higher Order Modes of a 3rd Harmonic Cavity with an Increased End-cup Iris", report TESLA-FEL-2003-01, DESY Hamburg (2003)

[7] I. Zagorodnov, T. Weiland and M. Dohlus, "Wakefields generated by LOLA-IV structure and the 3rd harmonic section in TTF-II", report TESLA-2004-01, DESY Hamburg (2004)

[8] K. Flöttmann, private communication, March 2004 